\title{
A novel SHARPIN-PRMT5-H3R2me1 axis is essential for lung cancer cell invasion
}

\author{
Tingxiong Fu ${ }^{1, *}$, Xiuwei Lv ${ }^{2, *}$, Qingzhi Kong ${ }^{3}$ and Changjing Yuan ${ }^{2}$ \\ ${ }^{1}$ Department of Pharmacy, The Central Hospital of Wuhan, Tongji Medical School, Huazhong University of Science and \\ Technology, Wuhan, Hubei, China \\ ${ }^{2}$ Department of Oncology of Integrated Traditional Chinese and Western Medicine, The Central Hospital of Wuhan, Tongji \\ Medical School, Huazhong University of Science and Technology, Wuhan, Hubei, China \\ ${ }^{3}$ The Central Hospital of Wuhan, Tongji Medical School, Huazhong University of Science and Technology, Wuhan, Hubei, China \\ *These authors contributed equally to this work
}

Correspondence to: Changjing Yuan, email: Changjing_Yuan@163.com

Keywords: SHARPIN, PRMT5, cancer invasion, histone modification, gene expression

Received: May 16, $2017 \quad$ Accepted: June 16, $2017 \quad$ Published: July 04, 2017

Copyright: Fu et al. This is an open-access article distributed under the terms of the Creative Commons Attribution License 3.0 (CC BY 3.0 ), which permits unrestricted use, distribution, and reproduction in any medium, provided the original author and source are credited.

\section{ABSTRACT}

SHARPIN (Shank-associated RH domain interacting protein) is the main component of the linear ubiquitin chain activation complex (LUBAC). SHARPIN is involved in regulating inflammation and cancer progression. However, whether SHARPIN plays an important role in lung cancer metastasis and the potential underlying mechanism are still unknown. Here, for the first time, we reported that SHARPIN expression is closely related to lung cancer progression. Moreover, SHARPIN plays a central role in controlling lung cancer cell metastasis. Mechanistic studies further revealed that PRMT5 (Protein arginine methyltransferase 5), responsible for catalyzing arginine methylation on histones, is a novel cofactor of SHARPIN. This finding provides the basis for further study of the crosstalk between protein ubiquitination and histone methylation. We further found that SHARPIN-PRMT5 is essential for the monomethylation of histones of chromatins at key metastasis-related genes, defining a new mechanism regulating cancer invasion. A novel MLL complex (ASH2 and WDR5) was implied in the link between histone arginine2 monomethylation (H3R2me1) and histone lysine4 trimethylation (H3K4me3) for the activation of metastasis-related genes. These novel findings establish a new epigenetic paradigm in which SHARPIN-PRMT5 has distinct roles in orchestrating chromatin environments for cancer-related genes via integrating signaling between H3R2me1 and H3K4me3.

\section{INTRODUCTION}

Lung cancer is one of the leading lethal cancers worldwide. However, the underlying mechanisms of this deadly cancer remain largely unknown $[1,2]$. Better elucidation of the pathologic mechanisms involved in lung cancer metastasis will enable the development of better treatment options for patients.

SHARPIN (Shank-associated RH domain interacting protein) is the main component of the linear ubiquitin chain activation complex (LUBAC), which gives rise to linear-type ubiquitin chains via linkages between methionine 1 and glycine 76 in proteins [3, 4]. LUBAC has been found to add a linear polyubiquitin chain to IkB kinase (IKK) complex to activate nuclear factor- $\mathrm{kB}$ (NF$\kappa B)$ signaling. Linear ubiquitination is also involved in the regulation of inflammation and immune signaling [5].

Recently, an increasing amount of evidence has demonstrated that SHARPIN is closely associated with oncogenesis. SHARPIN expression is frequently high in multiple human cancer types, including liver, ovarian, prostate, and breast cancer. SHARPIN plays tumorassociated roles during cancer biogenesis by promoting cell survival, growth, and invasion [6-10].

To explore the pathologic role of SHARPIN in regulating cancerogenesis, in the present study, we report 
for the first time that SHARPIN expression is upregulated in lung cancer. Moreover, we found PRMT5 as a novel cofactor of SHARPIN in regulating cancer-related gene expression. PRMT5 belongs to the protein arginine methyltransferase (PRMT) family, which transfers methyl groups from S-adenosylmethionine (SAM) to the guanidine nitrogen of arginine residues in proteins. PRMT5 is the main writer of symmetric dimethylarginine (Rme2s) and monomethylarginine (Rme1) [11-12]. Arginine methylation in histones is a common post-translational modification (PTM) of histones. Alterations in epigenetic regulation via the PTM of histones are highly correlated with cancer etiology and can lead to metastasis. Modulation the PTM of histones in chromatins is a key mechanism used by cancer cells to regulate transcription [13-14].

Here, we sought to determine how SHARPINPRMT5 interaction with its broader chromatin niche contributes to its ability to regulate cancer-related gene transcription. We demonstrate that the interaction with SHARPIN with a novel cofactor PRMTA5 plays biological roles in tumor progression and invasion via regulating a unique histone H3R2 methylation-coupled transcriptional activation in lung cancer cells.

\section{RESULTS}

\section{SHARPIN controls invasive phenotype of lung cancer cells}

To determine the correlation between human lung cancer and SHARPIN expression, RNA-sequencing results from the Cancer Genome Atlas (TCGA) were systematically mined. Interestingly, elevated SHARPIN expression was found in both human lung adenocarcinoma and squamous cell carcinoma compared with that in normal lung samples (Figure 1A). This exciting observation was also supported by results showing that SHARPIN RNA and protein expression is increased in several common lung cancer cell lines compared with those in normal lung cells (Figure 1B). To explore the role of SHARPIN alteration in cancer cells, migration and invasion assays were then performed to characterize changes in the mobility of SHARPIN-depleted cells. Immunoblots for SHARPIN confirmed efficient knockdown of SHARPIN in different lung cancer cells including A549, H460 and SK-MES-1 cells (Figure 1C). Remarkably, cell migration and invasion rates were significantly decreased in A549 (Figure 1D), H460 (Figure 1E) and SK-MES-1 cells (Figure 1F) with the depletion of SHARPIN, compared to those in control cells. These results suggested that SHARPIN plays an essential role in controlling the mobility of lung cancer cells.

\section{PRMT5 identified as a new cofactor that interacts with SHARPIN}

To explore the mechanism by which SHARPIN regulates lung cancer cell invasion, we attempted to identify new potential cofactors interacting with SHARPIN. Strikingly, by using LC-MS/MS, we detected several proteins in the SHARPIN immunoprecipitation fractions from A549 cells (Figure 2A). As indicated, a novel histone arginine methyltransferase, PRMT5, was identified as a potential cofactor of SHARPIN. Since PRMT5 is also widely involved in the pathologic progress of cancer metastasis, we tested if PRMT5 was a target of SHARPIN. With immunoprecipitation, we confirmed that SHARPIN interacts with PRMT5. As indicated in Figure 2B, immunoprecipitation was performed with antiSHARPIN antibody, and immunoblotting with PRMT5 proved the interaction between SHARPIN and PRMT5 (Figure 2B). Conversely, a similar conclusion was drawn from Figure 2C. Thus, we further questioned whether PRMT5 as a novel cofactor of SHARPIN also plays an important role in the invasion of cancer cells.

\section{PRMT5 mediates invasive phenotype of lung cancer cells}

Similar to the observations regarding alterations in SHARPIN expression in cancer (Figure 1A), the expression level of PRMT5 was also found to be dramatically elevated in both human lung adenocarcinoma and squamous cell carcinoma compared with that in normal lung samples by analysis of RNA-sequencing from TCGA (Figure 3A). Meanwhile, compared to normal lung cells, lung cancer cells displayed much higher levels of PRMT5 RNA and protein (Figure 3B). shRNA was then used to independently knock down PRMT5 in these lung cancer cell lines (Figure 3C). Knockdown of PRMT5 in A549 (Figure 3D), H460 (Figure 3E) and SK-MES-1 cells (Figure 3F) resulted in significantly reduced rates of migration and invasion compared to those in control cells. These results indicate that PRMT5 is necessary for maintaining malignancy-related cellular properties, including migration and invasion.

\section{SHARPIN regulates PRMT5-dependent arginine methylation of histones $\mathrm{H} 3$ and $\mathrm{H} 4$ concomitant with metastasis-related gene expression}

We hypothesized that SHARPIN influences local chromatin and gene expression by regulating PTM of PRMT5-enriched histones. We immunoblotted whole cell lysates from SHARPIN-knockdown A549 cells (Figure 4A). Compared with control, SHARPIN knockdown dramatically decreased PRMT5 protein expression. By monitoring the PTM of each histone associated with SHARPIN in SHARPIN-depleted IMR90 cells, we further demonstrated that SHARPIN specifically promotes methylation at H3R2me1 and H4R3me2s (Figure 4A).

Remarkably, SHARPIN knockdown also altered Epithelial-MesenchymalTransition (EMT) protein markers exhibited by the loss of expression of Vimentin and Snail (Figure 4A), consistent with the loss of invasive phenotypes 
A

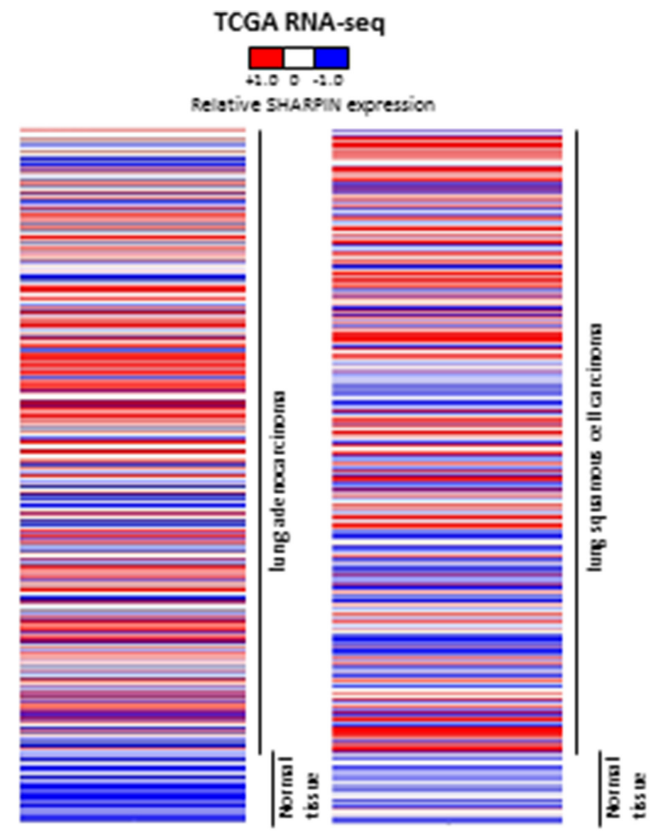

B
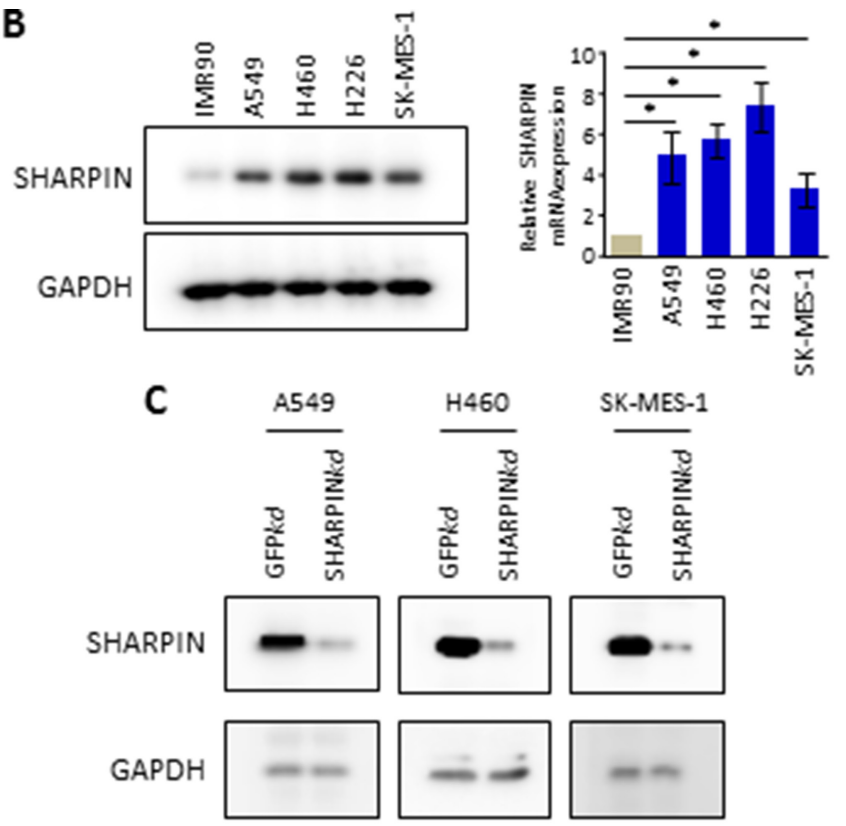
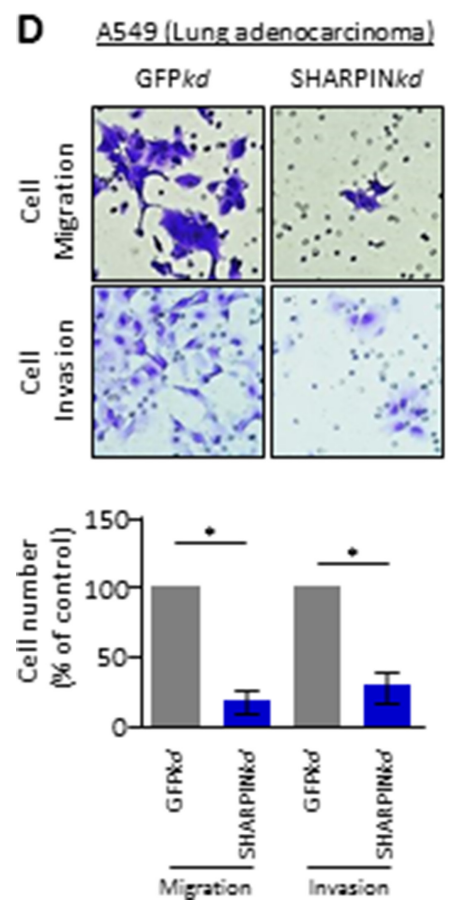

$\mathbf{E}$
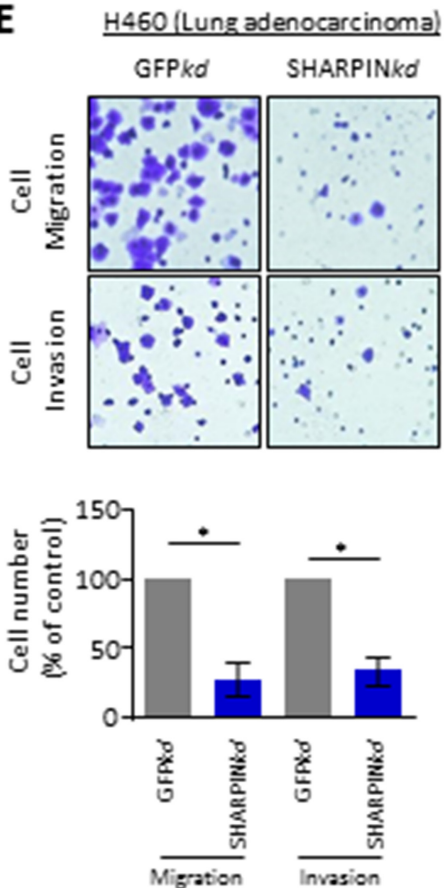

F SK-MES-1 (Lung squamouscell carcinoma)
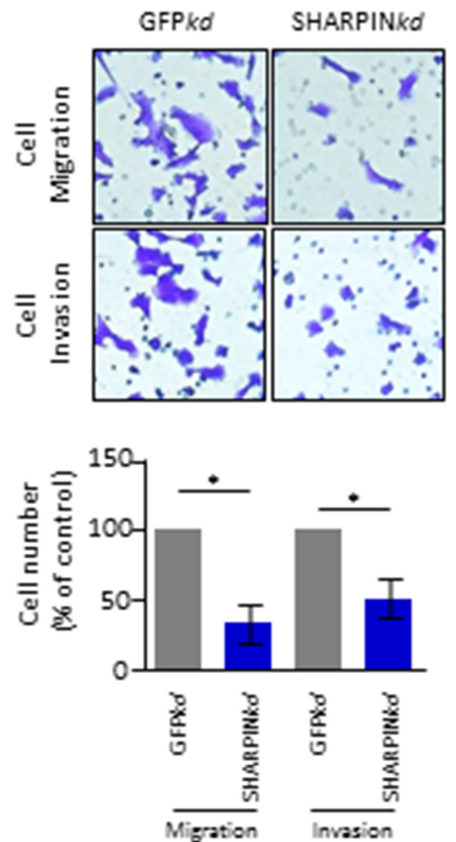

Figure 1: SHARPIN is a novel regulator of cell mobility in lung cancer cells. (A) Heat map depicting gene expression of SHARPIN from RNA-Sequencing data with lung adenocarcinoma (LUAD, normal tissues vs tumor tissues) or squamous cell carcinoma (LUSC, normal tissues vs tumor tissues) in The Cancer Genome Atlas (TCGA). Expressions of SHARPIN are ranked by sample types. RNA-Seq values are shown by indicated bar. (B) SHARPIN expression profile in lung normal fibroblast cells and lung cancer cell lines. (Left panel) Whole cell lysate immunoblots for SHARPIN and GAPDH (loading control). (Right panel) qRT-PCR for relative mRNA levels of SHARPIN. $\beta$-Actin was used as an internal control. Values are means \pm S.E.M. of three independent experiments. ${ }^{*} p<0.05$ from one-way ANOVA test. (C) Confirmation of depletion of SHARPIN in lung cancer A549, H460 and SK-MES-1 cell lines. Immunoblots for SHARPIN and GAPDH as control, from indicated cells infected with shRNA targeted against negative control (NC) or against SHARPIN. (D-F) Cell migration and invasion assays for lung cancer cell line (D) A549, (E) H460 and (F) SK-MES-1 with depletion of GFP (ctr) or SHARPIN. Representative crystal violet staining of invaded cells (upper panel) through a phase-contrast microscope (20X) is shown. Quantification of the invaded cells is shown (down panel). Values are mean \pm S.E.M. of three independent experiments. ${ }^{*} p<0.05$ from one-way ANOVA test. 
in the SHARPIN-depleted A549 cells (Figure 1D). Candidate metastasis-related genes (VIM, SNAI1, SNAI2 and CDH2) were identified from the literature $[14,15]$. By performing qRT-PCR experiments on SHARPIN-depleted A549 cells, we confirmed that the expression of metastasis-related genes is regulated by SHARPIN and PRMT5. As shown in Figure $4 \mathrm{~B}$, these genes were significantly reduced in expression after knockdown of either SHARPIN or PRMT5.

Since PRMT5 is also required for mediating the molecular consequences of EMT [14], these observations strongly suggested that SHARPIN is essential for maintaining PRMT5-associated chromatin environments in lung cancer A549 cells, with the activation of expression of cancer metastasis-related genes.

\section{SHARPIN is essential for PRMT5-mediated H3 arginine methylation in regulating the expression of cancer metastasis-related genes}

Since the invasive phenotypes, metastasis-related gene expression and PRMT5-dependent histone PTM of lung cancer cells were directly dependent on SHARPINPRMT5, this interaction likely alters the transcriptional outcomes by targeting histones. To test whether SHARPIN maintains the influence of PRMT5 on metastasis-related gene expression by targeting PTM of histones, we performed chromatin immunoprecipitation (ChIP) of promoter elements $(-1 \mathrm{~kb}$ to $+1 \mathrm{~kb})$ of these genes in A549 cell lines depleted of SHARPIN.

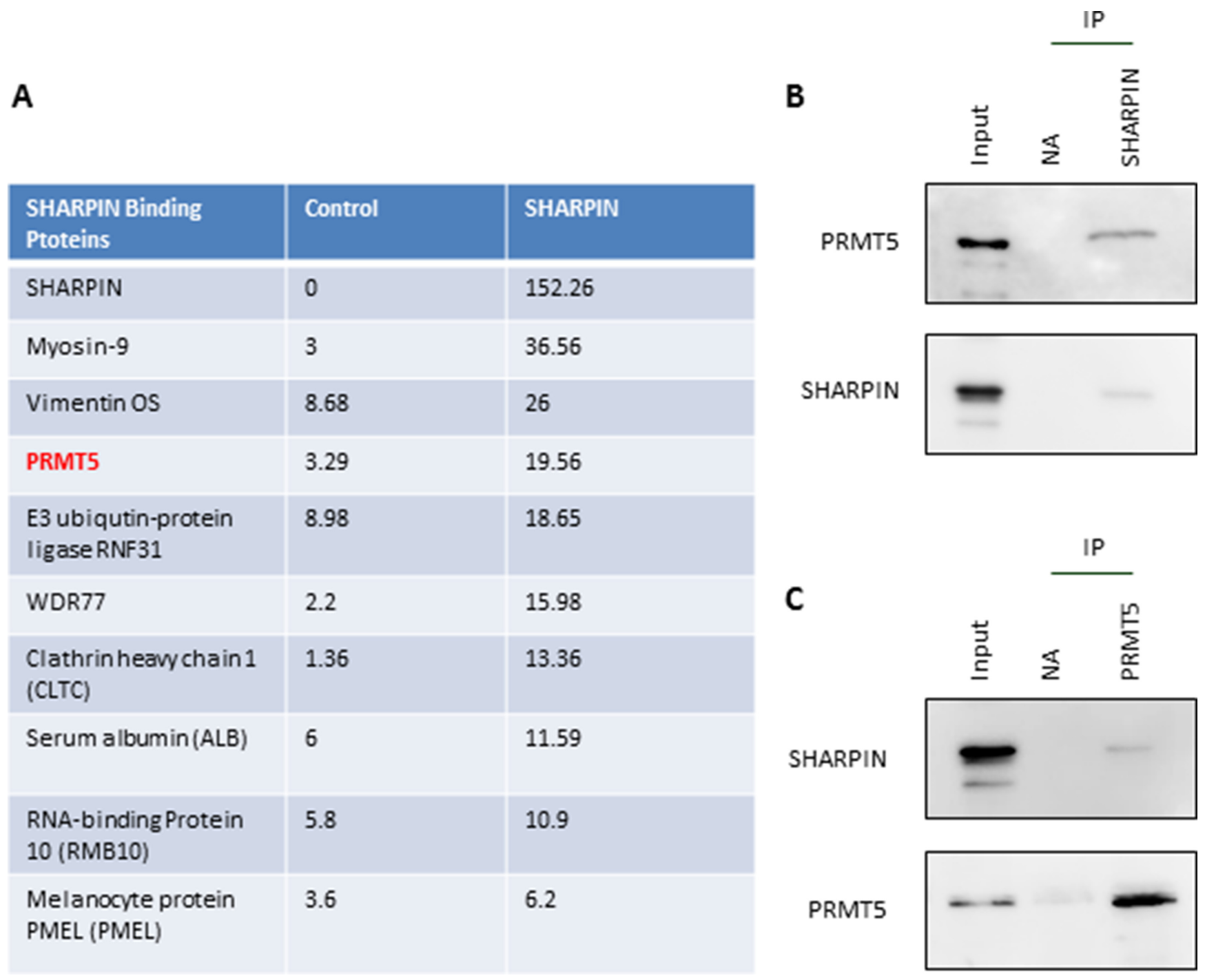

Figure 2: PRMT5 is a new cofactor that interacts with SHARPIN. (A) LC-MS/MS was performed to identify proteins that interact with SHARPIN in A549 cells. Top 10 functional annotations interacted with SHARPIN were listed. (B) SHARPIN Immunoprecipitation (IP) was performed in A549 cell lysates with antibodies directed against SHARPIN or no antibody (NA) as a control. Immunoblots were performed for both PRMT5 and SHARPIN (positive control). (C) PRMT5 Immunoprecipitation (IP) was performed in A549 cell lysates with antibodies directed against SHARPIN or no antibody (NA) as a control. Immunoblots were performed for both SHARPIN and PRMT5 (positive control). 
A

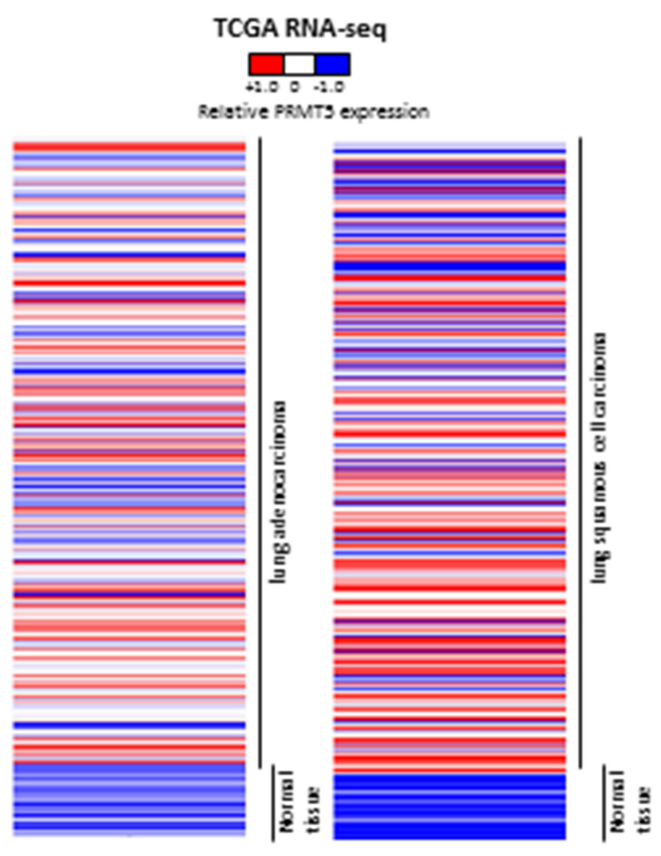

B

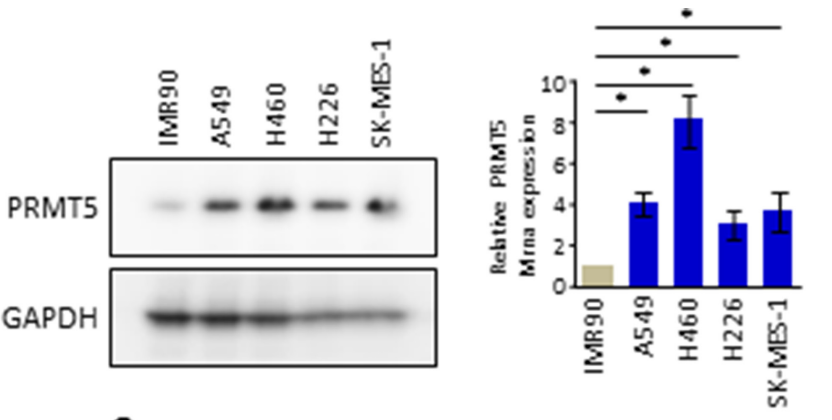

C

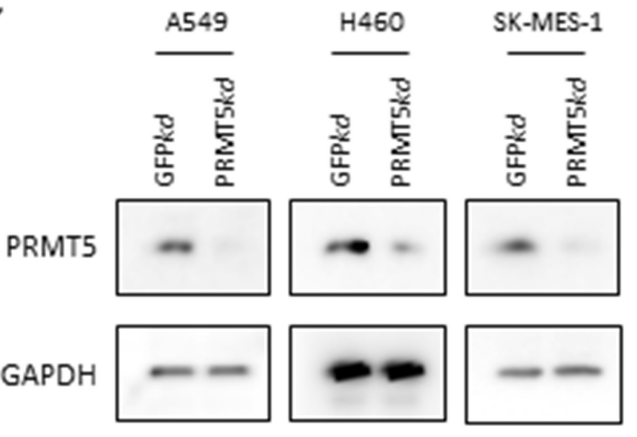

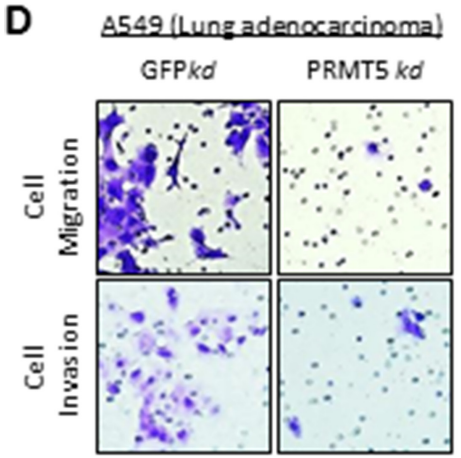

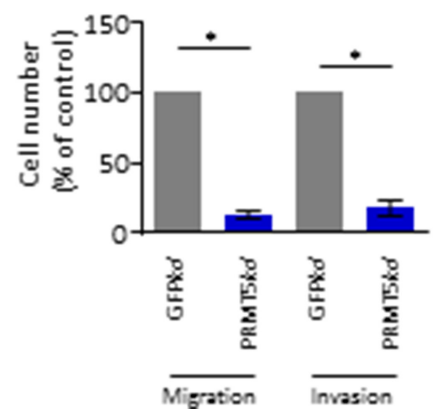

E
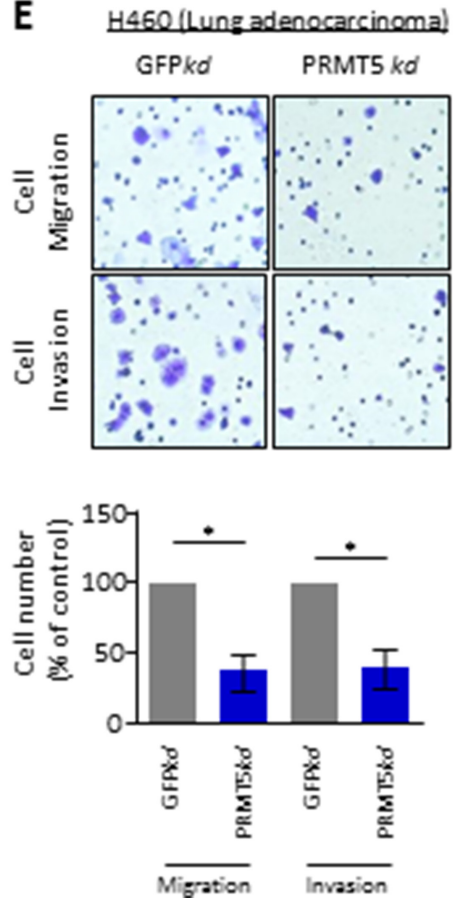

F SK-MES-1 (Lungsquamouscell carcinoma)
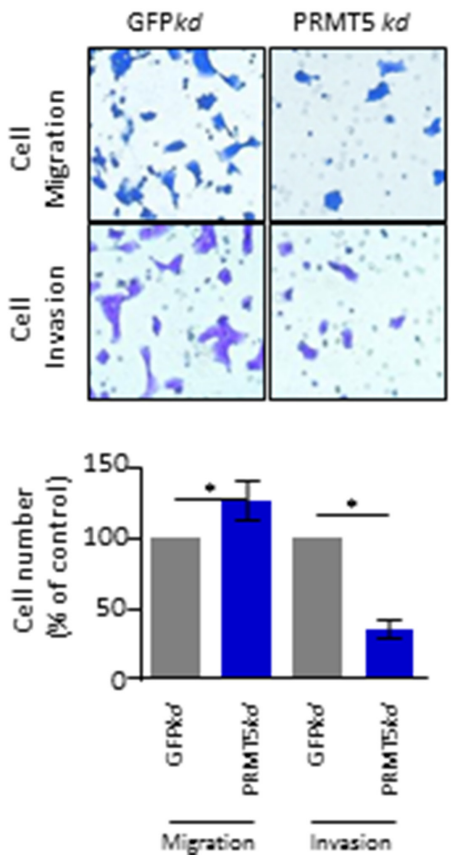

Figure 3: PRMT5 blocked lung cancer cell migration and invasion. (A) Heat map depicting gene expression of PRMT5 from RNA-Sequencing data with lung adenocarcinoma (LUAD, normal tissues vs tumor tissues) or squamous cell carcinoma (LUSC, normal tissues vs tumor tissues) in The Cancer Genome Atlas (TCGA). Expressions of PRMT5 are ranked by sample types. RNA-Seq values are shown by indicated bar. (B) PRMT5 expression profile in lung normal fibroblast cells and lung cancer cell lines. (Left panel) Whole cell lysate immunoblots for PRMT5 and GAPDH (loading control). (Right panel) qRT-PCR for relative mRNA levels of SHARPIN. $\beta$-Actin was used as an internal control. Values are means \pm S.E.M. of three independent experiments. ${ }^{*} p<0.05$ from one-way ANOVA test. (C) Confirmation of depletion of PRMT5 in lung cancer A549, H460 and SK-MES-1 cell lines. Immunoblots for PRMT5 and GAPDH as control, from indicated cells infected with shRNA targeted against negative control (NC) or against SHARPIN. (D-F) Cell migration and invasion assays for lung cancer cell line (D) A549, (E) H460 and (F) SK-MES-1 with depletion of GFP (ctr) or PRMT5. Representative crystal violet staining of invaded cells (upper panel) through a phase-contrast microscope (20X) is shown. Quantification of the invaded cells is shown (down panel). Values are mean \pm S.E.M. of three independent experiments. $* p<0.05$ from one-way ANOVA test. 
We systematically ChIPed all of the known PRMT5dependent PTMs including H3R2me1, H3R2me2s, H3R8me2s H4R3me1, and H4R3me2s. The asymmetric mark H4R3me2a was also tested as a negative control for PRMT5 activity (Figure 5A). The control marker
H3 was pulled down evenly (Figure 5B). As indicated by the heatmap (Figure 5A), metastasis-related genes were strikingly enriched in H3R2me1, H4R3me2a and H4R3me2s, while the observed enrichment of H3R2me1 was significantly decreased in the SHARPIN-knockdown

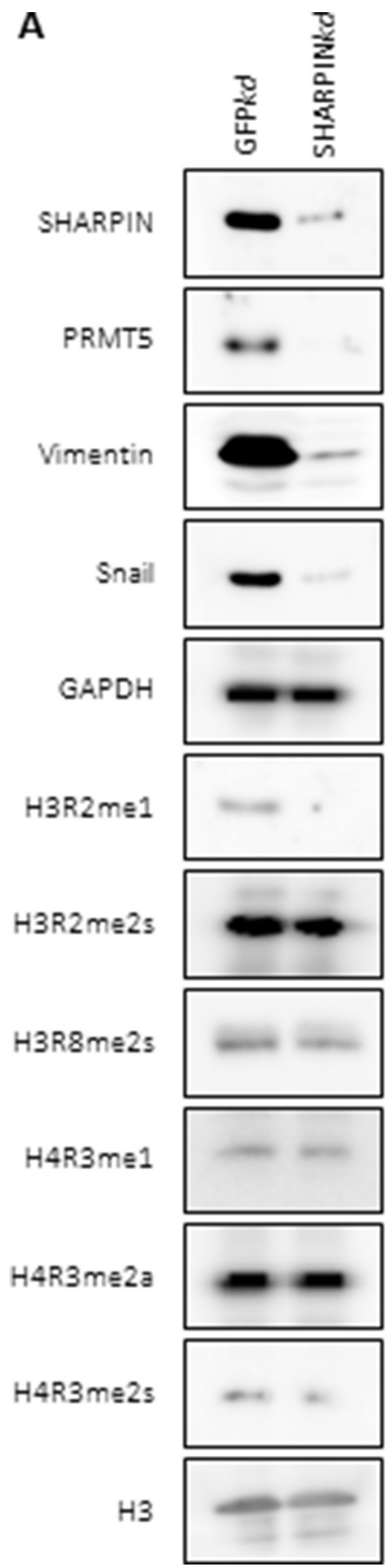

B

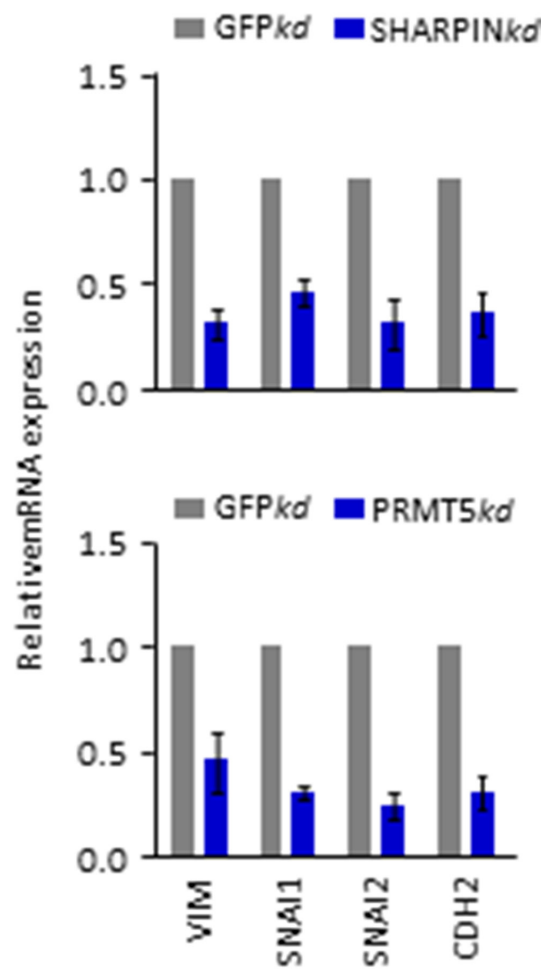

Figure 4: SHARPIN is necessary for PRMT5 and unique histone methylation concomitant with regulation of lung metastasis-related gene expression. (A) Immunoblots for SHARPIN, PRMT5, metastasis activator genes, and various histone methylarginine PTMs. GAPDH is used as a loading control. The tested A549 cells expressed shRNA targeted against GFP as a control $(\mathrm{GFP} k d$ ) or against SHARPIN (SHARPINkd). (B) Relative mRNA levels of indicated metastasis activator genes in A549 cells with depletion of GFP (ctr) vs SHARPIN (upper panel) or PRMT5 (down panel). mRNA was determined by qRT-PCR $\beta$-Actin was used as an internal control. Values are means \pm S.E.M. of three independent experiments. $p$ value is measured with one-way ANOVA test. 
cells. The ChIP-qPCR profile of all these histone PTMs supports the idea that SHARPIN is essential for PRMT5 in maintaining unique $\mathrm{H} 3$ arginine methylation (H3R2me1), which surrounds the promoter regions of cancer metastasis-related genes.

To highlight this novel gene regulation mechanism, we graphed the ChIP-qPCR values for various histone PTMs on VIM (-1 kb) (Figure 5C). As illustrated, among all the tested histone PTMs, H3R2me1, H4R3me2a and H4R3me2s were enriched at the VIM (-1 kb) locus in A549 cells. Surprisingly, with the depletion of SHARPIN, only the distribution of H3R2me1 on this region was significantly decreased.

Taken together, these findings provide insights into the mechanism by which SHARPIN maintains the expression of metastasis-related genes through PRMT5dependent H3R2me1 arginine methylation of histone H3.

\section{SHARPIN-PRMT5 promotes a characteristic chromatin environment in metastasis-related genes}

PRMT5-dependent H3R2me1 has been reported to enhance recruitment of the MLL complex for promoting H3K4me3 [14], which acts as a classic gene activating histone marker. We probed the possibility that SHARPIN might be crucial for the PRMT5-mediated epigenetic regulation of gene expression. Using ChIP assay, we confirmed that the knockdown of either SHARPIN or PRMT5 significantly reduced the abundance of H3K4me3 on VIM $(-1 \mathrm{~kb})$. With re-ChIP assay, we identified for the first time, the interaction between PRMT5 with all the known components of the MLL complex including ASH2, WDR5, HCFC1 and RBBP5 in A549 cells. The results demonstrated a strong interaction between PRMT5 and ASH2 or WDR5 on the regulatory region $(-1 \mathrm{~kb})$ of the VIM gene (Figure 6B). Strikingly, knockdown of SHARPIN dramatically reduced the occupancy of PRMT5 and ASH2 or WDR5 on the same tested region (Figure 6C). These novel findings demonstrate that SHARPIN plays a key role in incorporating PRMT5 into an activating MLL complex (ASH1 and WDR5), which mediates H3K4m3 to regulate the expression of metastasis-related genes.

In a detailed model (Figure 6D), our findings suggest that SHARPIN is responsible for mediating the expression of cancer metastasis-related genes, which leads to changes in the mobility of cancer cells. Meanwhile, PRMT5 is identified as a novel cofactor of SHARPIN, which targets the methylation of histone $\mathrm{H} 3$ arginine 2 for gene activation through recruitment of the MLL complex (ASH2 and WDR5) to promote H3K4me3.

\section{DISCUSSION}

SHARPIN, a multifunctional molecule, participates in a variety of biological processes, including activation of nuclear factor- $\mathrm{B}$ signaling and inhibition of tumor suppressor genes [3-5]. SHARPIN has been implicated in the pathologic progression in several cancers. Here, we identified that SHARPIN is closely related to lung cancerogenesis. We further showed that SHARPIN plays a novel role in lung cancer metastasis. SHARPIN is mainly localized in the cytoplasm of cancer cells; however, a fraction of SHARPIN is also localized in the nucleus $[16,17]$, which implies that SHARPIN can interact with specific transcription factors to regulate gene expression. Nevertheless, there stands a long-time puzzle regarding the specific molecule that can integrate SHARPIN signaling with chromatins for the regulation of gene expression. Identification of PRMT5 as a novel cofactor of SHARPIN in our study provided a key to solve this problem.

Although a wealth of literature has supported the essential biological functions of PRMT5 in targeting histones [11, 14, 18], including symmetric dimethylation of histones $\mathrm{H} 2 \mathrm{~A}$ and $\mathrm{H} 4$ on $\mathrm{R} 3$ or histone $\mathrm{H} 3$ on R8 and asymmetric methylation of H3 on R2, the exact type of histone arginine methylation mediated by SHARPINPRMT5 in lung cancer metastasis is still unknown. In this study, we hypothesized that SHARPIN-PRMT5 forms a crucial intermediate in relaying cytoplasmic signaling pathways to chromatins by altering the PTM of histones to control transcription. In this study, we found that SHARPIN activates PRMT5 to specifically target histone H3R2 for monomethylation, which is responsible for the subsequent activation of cancer-related genes.

Histone post-translational modifications (PTMs) are important for proliferation and differentiation of cancer cells, which play a central role in the etiologic and pathologic mechanisms of cancer [19, 20]. Our data indicate that SHARPIN-PRMT5 has distinct roles in orchestrating chromatin environments for cancerrelated genes by maintaining the monomethylation of histone $\mathrm{H} 3$ arginine and trimethylation of histone lysine 4. H3R2me2s has been found to have the capacity to recruit the WDR5 protein, which promotes $\mathrm{H} 3 \mathrm{~K} 4 \mathrm{me} 3$ to regulate gene expression through its associated MLL lysine methyltransferase $[18,21]$. The similarity of the structure of H3R2me1 to that of H3R2me2s suggests that H3R2me1 may also recruit WDR5 through a similar mechanism. In our study, for the first time, we systematically screened the known components of the MLL methyltransferase complex to identify the ones binding to H3R2me1. The exciting results exceeded our expectation, and we speculated that there was a strong interaction between SHARPIN-PRMT5-dependent H3R2me1 and ASH2 or WDR5, which forms the activating MLL complex to mediate $\mathrm{H} 3 \mathrm{~K} 4 \mathrm{~m} 3$ to regulate the expression of metastasisrelated genes. These results provide insights into the epigenetic mechanism by which SHARPIN-PRMT5 is involved in cancer metastasis. It is worthwhile to further explore whether this novel epigenetic mechanism is also involved in other types of cancers metastasis. 
In conclusion, we demonstrated a new SHARINPRMT5-H3R2me1 axis mediating metastasis in invasive lung cancer cells. As new diagnostic molecules and new drug candidates are required for personalized treatment for lung cancer metastasis, it is essential to have more insights into epigenetic gene regulatory pathways. Ubiquitination and methylation are two important posttranslational modifications of proteins in human cells,
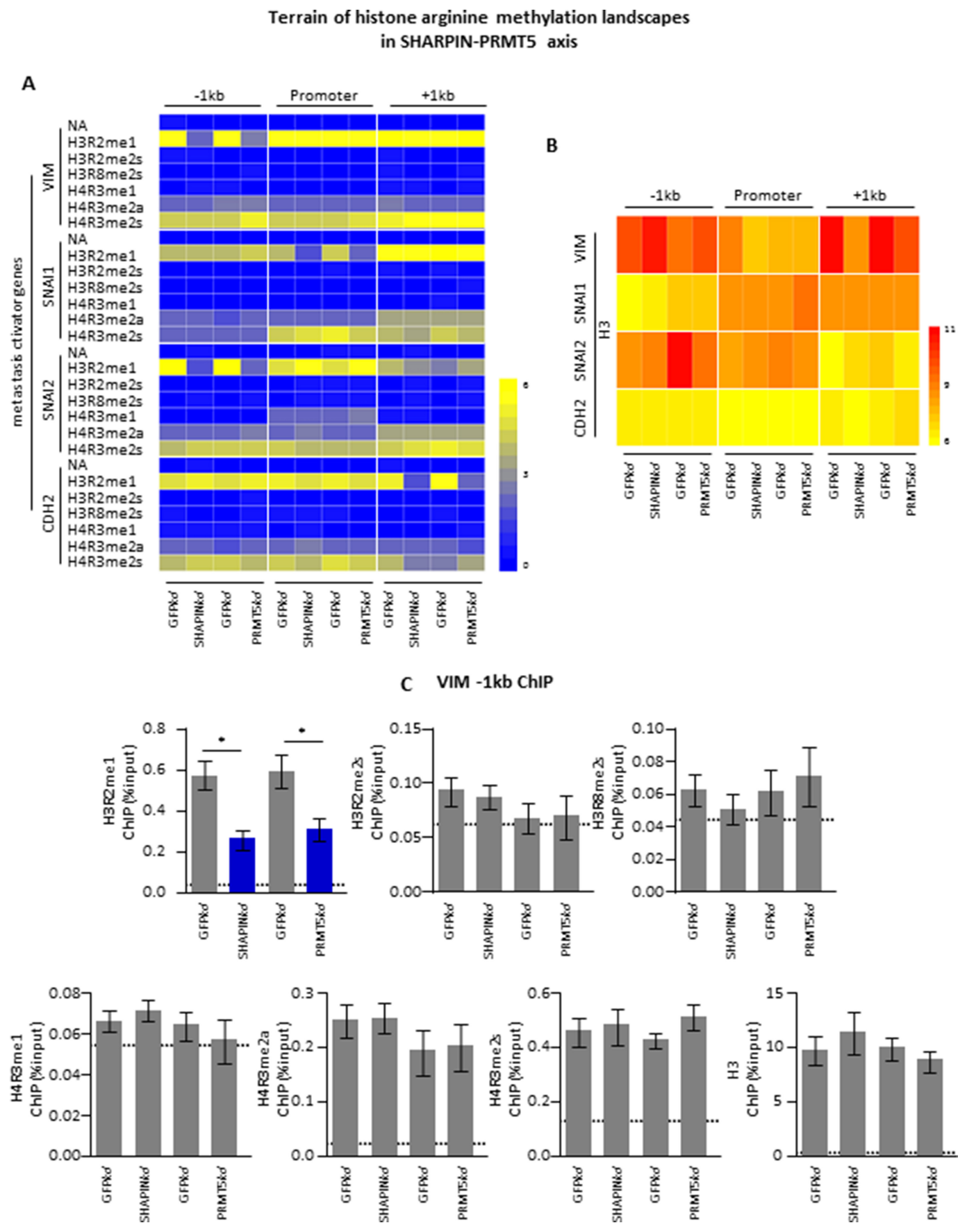

Figure 5: SHARPIN-PRMT5 controls a unique histone methylarginine code to regulate metastasis-related gene expression. (A) The values of ChIP-qPCR from PRMT5 and various histone methylarginines is used to generated a heatmap to demonstrate the characteristic distribution of histone methylarginines surrounding the candidate genes at $-1 \mathrm{~kb}$, the promoter, or $+1 \mathrm{~kb}$ regions. The heatmap was arrayed from blue (no enrichment) to yellow (maximal enrichment) as shown by indicated bar. (B) Histone H3 is used as control for ChIP experiments (A). Heatmap generated by ChIP-qPCR values for histone $\mathrm{H} 3$ values at $-1 \mathrm{~kb}$, the promoter, or $+1 \mathrm{~kb}$ of the metastasis activator genes. The results indicated the $\mathrm{H} 3$ was pulled down evenly. There was no significant $\mathrm{H} 3$ enrichment changes between different groups. $p$ value is measured with one-way ANOVA test. (C) Illustration of ChIP-qPCR results for VIM (-1kb) indicated in (A and B). The horizontal dotted line indicated the upper limit of the $95 \%$ confidence interval of the signal from no-antibody (NA) control ChIPs. Values are mean \pm S.E.M. of three independent experiments. ${ }^{*} P<0.05$ from one-way ANOVA test. 
which are widely involved in the spatial and temporal regulation of gene expression [22-26]. Although we have a wealth of knowledge on ubiquitination and methylation as individual modifications, the extent to which the two modifications orchestrate to regulate gene expression in the context of different cellular signaling in cancer cells is poorly understood. Therefore, it is important to determine how the epigenetic landscape is further coordinated by

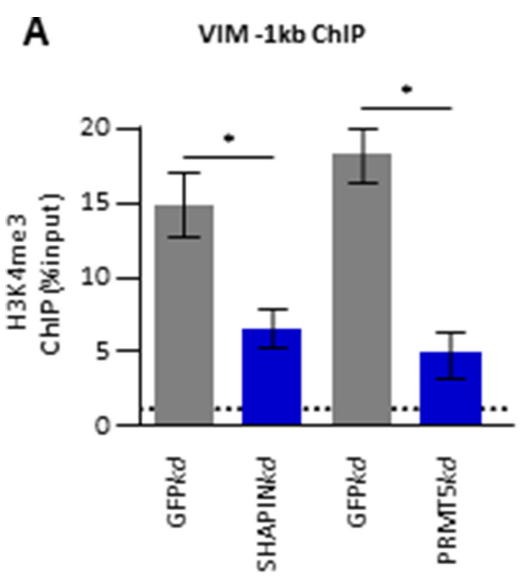

B

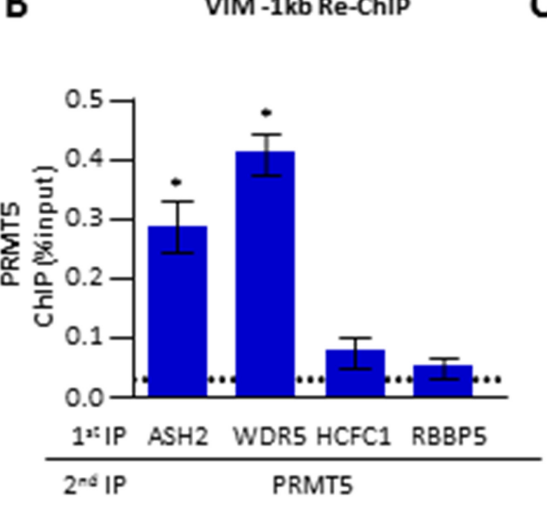

C

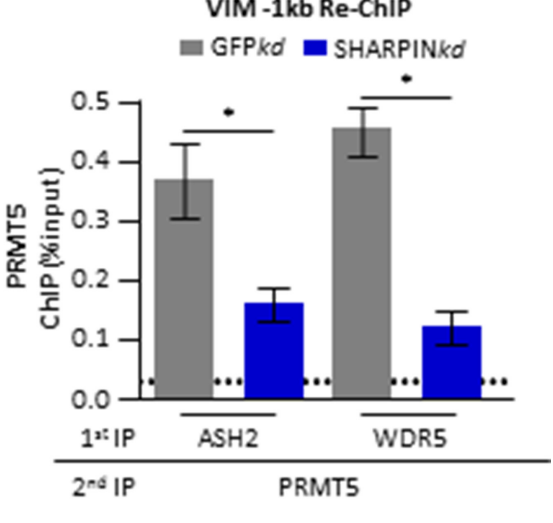

D
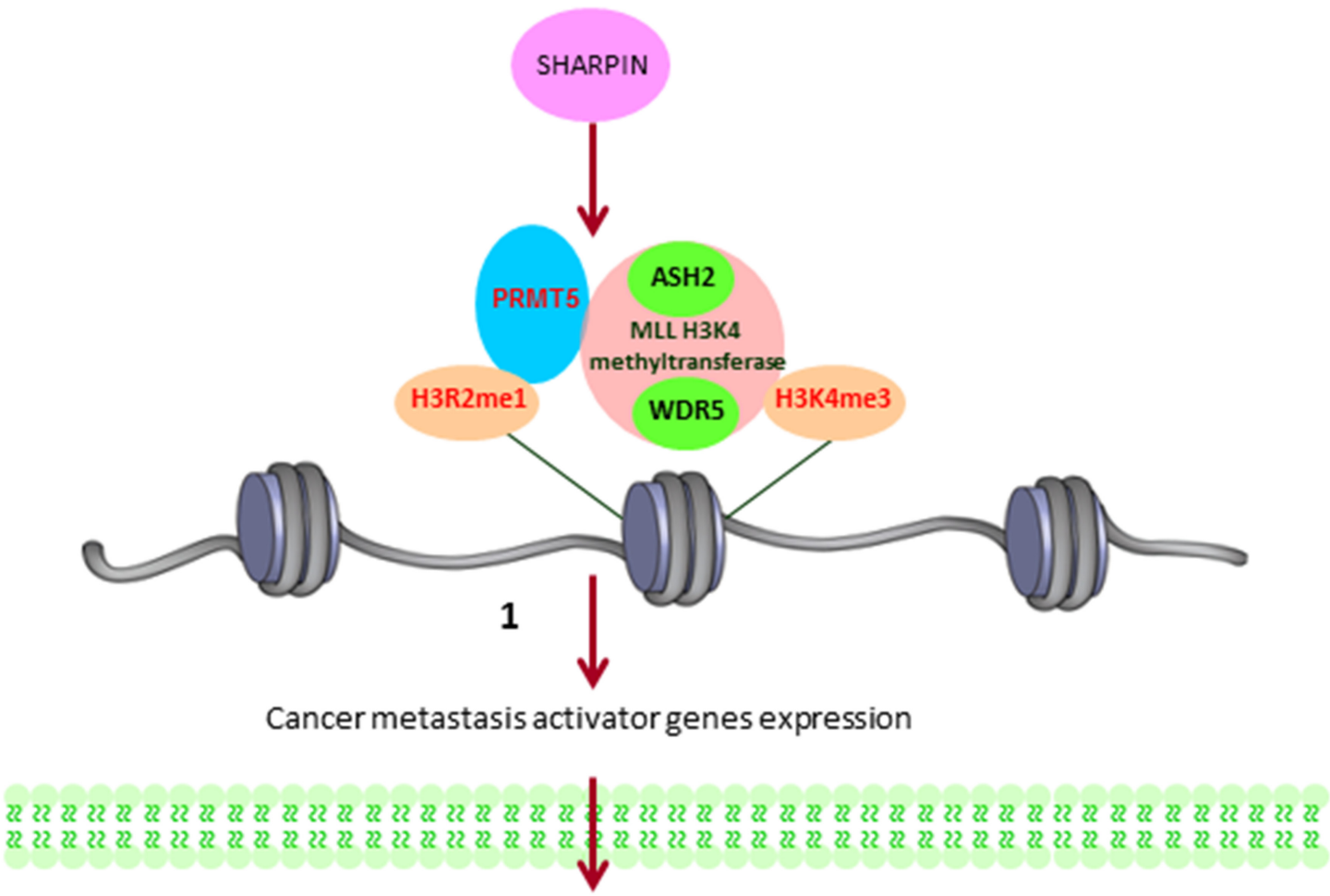

\section{Lung cancer cell invasion}

Figure 6: A novel SHARPIN-PRMT5-H3R2me1 axis is involved in regulating of metastasis-related gene expression. (A) H3K4me3 and H3 (control) ChIP-qPCR experiments of Vim (-1kb) in A549. The tested A549 cells expressed shRNA targeted against GFP as a control (GFP $k d$ ), against SHARPIN (SHARPINkd) or against PRMT5 (PRMT5kd). (B) Re-ChIP was performed in A549 cells with $1^{\text {st }}$ round pull down of various MLL complex antibodies including ASH2, WDR5, HCFC1 or RBBP5 as well as 2nd round pull down of PRMT5 antibody. (C) A549 cells were depleted of GFP (ctr) or SHARPIN and harvested for Re-ChIP test. Re-ChIP was performed with 1st round pull down of ASH2 or WDR5 antibody as well as 2nd round pull down of PRMT5 antibody. (D) Model depicting SHARPIN affecting PRMT5 modulation of metastasis activator gene expression via H3Rme1 histone arginine methylation. 
ubiquitination and methylation in future investigations. Meanwhile, more efforts are needed to evaluate and improve the safety of these novel approaches to treat lung cancer metastasis in clinical trials.

\section{MATERIALS AND METHODS}

\section{Cell culture}

IMR90 primary human fetal lung fibroblast cells (ATCC) were cultured in MEM medium with 10\% FBS. Various lung cancer cells A549 (ATCC), H460 (ATCC), H226 (ATCC) and SK-MES-1 were cultured in RPMI medium with $10 \%$ FBS. Mycoplasma contamination was routinely tested in these cell lines. Stable depletion of SHARPIN, PRMT5 or GFP (control) in A549, H460 and SK-MES-1 were performed by retroviralmediated expression of shRNA with the pSuper.Retro system obtained from the TRC genome-wide shRNA collection. The SHARPIN shRNA targeting sequence was 5'- GGCUGCAGGUCACACUUGATT-3'. The PRMT5 shRNA targeting sequence was 5'- GCCCA GTTTGAGATGCCTTAT-3' [14]. The GFP (control) shRNA targeting sequence was 5'-TACAACA GCCACAACGTCTAT-3' [14]. The cells were selected and maintained under $0.5 \mathrm{mg} / \mathrm{ml} \mathrm{G} 418$.

\section{Cell migration and invasion assays}

Cell migration and invasion assays were performed in Falcon permeable supports for 24 well plates essentially according to the manufacturer's instruction (Corning, 353097). Cells migrated on the lower surface of the PET membrane were fixed with $4 \%$ paraformaldehyde for 15 min at room temperature, then stained with $0.2 \%$ crystal violet in $2 \%$ ethanol for $20 \mathrm{~min}$ at room temperature. The cells were imaged with a Nikon Diaphot phase contrast microscope. At least three separate microscopic fields were counted per membrane.

\section{Immunoblots and acid-extraction of histones}

Whole cell-lysis and acid-extraction of histones were performed as previously described [27]. Briefly, the $90 \%$ confluent cells in one $10 \mathrm{~cm}$ dish were lysed in $100 \mu \mathrm{l}$ of $0.1 \%$ Triton X-100 lysis buffer. After centrifugation at 14,000 r.p.m. for $10 \mathrm{~min}$ at $4^{\circ} \mathrm{C}$, the supernatant of the lysate was collected as the whole celllysis. The pellet was resuspended in $80 \mu \mathrm{l} 0.5 \mathrm{M} \mathrm{HCl}$ at $4^{\circ} \mathrm{C}$ for $2 \mathrm{~h}$ under agitation to liberate acid-extractable proteins. After centrifugation at 14,000 r.p.m. for 10 min at $4{ }^{\circ} \mathrm{C}$, the supernatant was neutralized with 20 $\mu 12 \mathrm{M}$ Tris. Immunoblots were performed on PVDF (Immobilon, Millipore) and anti-mouse or anti-rabbit secondary antibody was used for detection by ECL chemiluminescence according to the manufacturer's instructions (Lumigen, TMA-6). Then, immunoblots were imaged with the ImageQuant LAS4000 imaging system. A list of antibodies used is presented in Supplementary Table 1. All immunoblots were repeated at least twice with independent biological samples.

\section{Co-immunoprecipitation (Co-IP)}

Co-IP was performed essentially as previously described [28]. Cell lysates were pre-cleared with protein A-agarose beads at $4^{\circ} \mathrm{C}$ for $2 \mathrm{~h}$. The resulting supernatant was subsequently incubated at $4^{\circ} \mathrm{C}$ overnight with protein A-agarose beads reacted with either SHAPRIN rabbit antibody or PRMT5 rabbit antibody, while Noantibody (NA) was used as negative control. The immunoprecipitated proteins were analyzed by subjected to SDS-PAGE and western blotting. All immunoblots have been repeated at least twice with independent biological samples.

\section{RNA extraction and qRT-PCR}

RNA purification and qRT-PCR were performed as described previously [29]. Briefly, mRNA levels were analyzed by reverse transcription followed by quantitative PCR (qRT-PCR). RNA was isolated with Rneasy mini kit (Qiagen, 74104) according to the manufacturer's protocol. The RNA was reverse transcribed with Moloney murine leukemia virus (MMLV) reverse transcriptase (Invitrogen) and a dT18 primer. cDNA, SYBR Green PCR master mix (Roche 04707516001). The efficiency-corrected threshold cycle $(\triangle \mathrm{CT})$ method was used to determine the relative levels of RNA. Primer sequences used in this study are listed in Supplementary Table 2.

\section{Chromatin immunoprecipitation (ChIP) and Re- chromatin immunoprecipitation (Re-ChIP)}

Chromatin Immunoprecipitation (ChIP) was performed as previously described [29]. Briefly, cells were grown to $90 \%$ confluence in $15 \mathrm{~cm}$ dishes, cross-linked with $1 \%$ formaldehyde, and sonicated. After sonication, the cross-linked chromatins were immunopreciptated with ChIP-grade antibodies against PRMT5, histone $\mathrm{H} 3$ and various histone methylation modification including H3R2me1, H3R2me2s, H3R8me2s, H4R3me1, H4R3me2a and H4R3me2s. Noantibody (NA) controls were always included as negative control. The enrichment of the specific amplified region was detected by quantitative real-time PCR with SYBR Green (Roche 04707516001) and percentage enrichment of each immunoprecipitated material over input chromatin DNA was shown. qRT-PCR with SYBR was performed to detect the indicated regions of the target genes. For Re-Chromatin Immunoprecipitation (Re-ChIP) [30], immunoprecipitated complexes were eluted with the elution buffer ( $1 \%$ SDS, 
$100 \mathrm{mM} \mathrm{NaCO} 3)$, diluted with the re-ChIP buffer $(1 \%$ Triton X-100, 2 mM EDTA, $150 \mathrm{mM} \mathrm{NaCl}, 20 \mathrm{mM}$ Tris $\mathrm{pH} 8.1$ ), which was subjected to immunoprecipitation with PRMT5 antibody. Antibodies and primers used for ChIP-qPCR are shown in Supplementary Table 1 and Supplementary Table 3 respectively.

\section{Mass spectrometry}

The mass spectrometry was performed essentially as described previously [31]. 10 plates of $15 \mathrm{~cm}$ dishes of A549 cells were grown to $90 \%$ confluence. The cells were collected and lysed in lysis buffer $(150 \mathrm{mM} \mathrm{NaCl}, 50 \mathrm{mM}$ Tris- $\mathrm{HCl}, \mathrm{pH} 7.5,1 \%$ Triton $\mathrm{X}-100$ and and $1 \times$ protease inhibitor cocktail). The lysates were precleared with protein A-agarose beads at $4^{\circ} \mathrm{C}$ for $3 \mathrm{~h}$. The supernatant was used in immunoprecipitations at $4^{\circ} \mathrm{C}$ overnight with antibody against SHARPIN as indicated. No-antibody (NA) controls were always included. Then, the beads were washed with lysis buffer for 3 times at $4^{\circ} \mathrm{C}$. After centrifugation at $4^{\circ} \mathrm{C}$, the resulting beads were subjected to trypsin digestion. The supernatant with SHAPIN-binding proteins were detected by mass spectrometry ${ }^{19}$.

\section{Statistical analysis}

All immunoblots were independently performed at least three times. All migration and invasion assays, ChIP, Re-ChIP and RT-qPCR experiments were repeated at least three times with independent biological samples. All data are presented as means \pm s.e.m. One-way ANOVA test was used to determine the significance of differences between samples indicated in Figures.

\section{ACKNOWLEDGMENTS AND FUNDING}

We are grateful to current and former members of the Yuan laboratory for discussions. This work was supported by grants from Health and Family Planning Commission of Wuhan Municipality (Grant No. WZ14Z17).

\section{CONFLICTS OF INTEREST}

The authors declare no conflicts of interest.

\section{REFERENCES}

1. Jemal A, Siegel R, Xu J, Ward E. Cancer statistics. CA Cancer J Clin. 2010; 60:277-300.

2. Guzmán L, Depix MS, Salinas AM, Roldán R, Aguayo F, Silva A, Vinet R. Analysis of aberrant methylation on promoter sequences of tumor suppressor genes and total DNA in sputum samples: a promising tool for early detection of COPD and lung cancer in smokers. Diagn Pathol. 2012; 7:1596-1597.
3. Tokunaga F, Nakagawa T, Nakahara M, Saeki Y, Taniguchi M, Sakata S, Tanaka K, Nakano H, Iwai K. SHARPIN is a component of the NF-kappaB-activating linear ubiquitin chain assembly complex. Nature. 2011; 471:633-636.

4. Ikeda F, Deribe YL, Skånland SS, Stieglitz B, Grabbe C, Franz-Wachtel M, van Wijk SJ, Goswami P, Nagy V, Terzic J, Tokunaga F, Androulidaki A, Nakagawa T, et al. SHARPIN forms a linear ubiquitin ligase complex regulating NF- $\mathrm{KB}$ activity and apoptosis. Nature. 2011; 471:637-641.

5. Gerlach B, Cordier SM, Schmukle AC, Emmerich CH, Rieser E, Haas TL, Webb AI, Rickard JA, Anderton H, Wong WW, Nachbur U, Gangoda L, Warnken U, et al. Linear ubiquitination prevents inflammation and regulates immune signalling. Nature. 2011; 471:591-596.

6. Tanaka Y, Tateishi K, Nakatsuka T, Kudo Y, Takahashi R, Miyabayashi K, Yamamoto K, Asaoka Y, Ijichi H, Tateishi R, Shibahara J, Fukayama M, Ishizawa T, et al. Sharpin promotes hepatocellular carcinoma progression via transactivation of Versican expression. Oncogenesis. 2016; 12;5:e277.

7. Jung J, Kim JM, Park B, Cheon Y, Lee B, Choo SH, Koh SS, Lee S. Newly identified tumor associated role of human sharpin. Mol Cell Biochem. 2010; 340:161-167.

8. Zhang Y, Huang H, Zhou H, Du T, Zeng L, Cao Y, Chen J, Lai Y, Li J, Wang G, Guo Z. Activation of nuclear factor $\kappa \mathrm{B}$ pathway and downstream targets survivin and livin by sharpin contributes to the progression and metastasis of prostate cancer. Cancer. 2014; 120:3208-3218.

9. De Melo J, Tang D. Elevation of SIPL1 (sharpin) increases breast cancer risk. PLoS ONE. 2015; 10:e0127546.

10. Li J, Lai Y, Cao Y, Du T, Zeng L, Wang G, Chen X, Chen J, Yu Y, Zhang S, Zhang Y, Huang H, Guo Z. Sharpin overexpression induces tumorigenesis in human prostate cancer LNCaP, DU145 and PC-3 cells via nf- $\kappa$ B /ERK/Akt signaling pathway. Med Oncol. 2015; 32:444.

11. Stopa N, Krebs JE, Shechter D. The PRMT5 arginine methyltransferase: many roles in development, cancer and beyond. Cell Mol Life Sci. 2015; 72:2041-59.

12. Sarkar S, Horn G, Moulton K, Oza A, Byler S, Kokolus S, Longacre M. Cancer development, progression, and therapy: an epigenetic overview. Int J Mol Sci. 2013; 14:21087-21113.

13. Tam WL, Weinberg RA. The epigenetics of epithelialmesenchymal plasticity in cancer. Nat Med. 2013; 19: 1438-1449.

14. Chen H, Lorton B, Gupta V, Shechter D. A TGF $\beta$-PRMT5MEP50 axis regulates cancer cell invasion through histone $\mathrm{H} 3$ and $\mathrm{H} 4$ arginine methylation coupled transcriptional activation and repression. Oncogene. 2017; 36:373-386.

15. Liu J, Hu G, Chen D, Gong AY, Soori GS, Dobleman TJ, Chen XM. Suppression of SCARA5 by Snaill is essential for EMT-associated cell migration of A549 cells. Oncogenesis. 2013; 2:e73.

16. Rantala JK, Pouwels J, Pellinen T, Veltel S, Laasola P, Mattila E, Potter CS, Duffy T, Sundberg JP, Kallioniemi O, 
Askari JA, Humphries MJ, Parsons M, et al. Sharpin is an endogenous inhibitor of $\beta 1$-integrin activation. Nat Cell Biol. 2011; 13:1315-1324.

17. Jung J, Kim JM, Park B, Cheon Y, Lee B, Choo SH, Koh SS, Lee S. Newly identified tumor associated role of human sharpin. Mol Cell Biochem. 2010; 340:161-167.

18. Yuan CC, Matthews AG, Jin Y, Chen CF, Chapman BA, Ohsumi TK, Glass KC, Kutateladze TG, Borowsky ML, Struhl K, Oettinger MA. Histone H3R2 Symmetric Dimethylation and Histone H3K4 Trimethylation Are Tightly Correlated in Eukaryotic Genomes. Cell Reports. 2012; 1:83-90.

19. Migliori V, Müller J, Phalke S, Low D, Bezzi M, Mok WC, Sahu SK, Gunaratne J, Capasso P, Bassi C, Cecatiello V, De Marco A, Blackstock W, et al. Symmetric demethylation of H3R2 is a newly identified histone mark that supports euchromatin maintenance. Nat Struct Mol Biol. 2012; 19:136-144.

20. Esteller M. Cancer epigenomics: DNA methylomes and histone-modification maps. Nat Rev Genet. 2007; 8:286-98.

21. Dawson MA, Kouzarides T. Cancer epigenetics: from mechanism to therapy. Cell. 2012; 6:12-27.

22. Morrow JK, Lin HK, Sun SC, Zhang S. Targeting ubiquitination for cancer therapies. Future Med Chem. 2015; 7:2333-2350.

23. Dwane L, Gallagher WM, Ní Chonghaile T, O'Connor DP. The Emerging Role of Non-traditional Ubiquitination in Oncogenic Pathways. J Biol Chem. 2017; 292:3543-3551.

24. Tam WL, Weinberg RA. The epigenetics of epithelialmesenchymal plasticity in cancer. Nat Med. 2013; 19: 1438-1449.
25. Brien GL, Valerio DG, Armstrong SA. Exploiting the Epigenome to Control Cancer-Promoting Gene-Expression Programs. Cancer Cell. 2016; 11:464-476.

26. Han $\mathrm{M}, \mathrm{Xu} \mathrm{W}$, Cheng $\mathrm{P}$, Jin $\mathrm{H}$, Wang $\mathrm{X}$. Histone demethylase lysine demethylase $5 \mathrm{~B}$ in development and cancer. Oncotarget. 2017; 8:8980-8991. http://doi. org/10.18632/oncotarget.13858.

27. Chen H, Ruiz PD, McKimpson WM, Novikov L, Kitsis RN, Gamble MJ. MacroH2A1 and ATM play opposing roles in paracrine senescence and the senescence-associated secretory phenotype. Mol Cell. 2015; 59:719-731.

28. Zhao C, Matthews J, Tujague M, Wan J, Strom A, Toresson G, Lam EW, Cheng G, Gustafsson JA, DahlmanWright K. Estrogen receptor beta2 negatively regulates the transactivation of estrogen receptor alpha in human breast cancer cells. Cancer Res. 2007; 67:3955-3962.

29. Chen H, Ruiz PD, Novikov L, Casill AD, Park JW, Gamble MJ. MacroH2A1.1 and PARP-1 cooperate to regulate transcription by promoting CBP-mediated $\mathrm{H} 2 \mathrm{~B}$ acetylation. Nat Struct Mol Biol. 2014; 21:981-989.

30. Yang Y, Yuan Z, Gao Y, Zhou J, Chen Q, Xu Y. Myocardinrelated transcription factor A mediates OxLDL-induced endothelial injury. Circ Res. 2011; 108:797-807.

31. Brill LM, Motamedchaboki $\mathrm{K}$, Wu S, Wolf DA. Comprehensive proteomic analysis of Schizosaccharomyces pombe by two-dimensional HPLC-tandem mass spectrometry. Methods. 2009; 48:311-319. 